\title{
Research and Design of Automated Demand Response Terminals for Central Air Conditioning System
}

\author{
Zhao Zhiwen $^{1, \text { a }}$, Chen Songsong ${ }^{2, \mathrm{~b}}$, Han Wanjiao ${ }^{3, \mathrm{c}}$, Zhao Jianyi ${ }^{1}$, Zheng Jun ${ }^{1}$ \\ and He Ziheng ${ }^{4, d^{*}}$ \\ ${ }^{1}$ State Grid (Shanghai) Smart Power Grids R\&D Investment CO.LTD, Shanghai, China \\ ${ }^{2}$ China Electric Power Research Institute, Beijing, China \\ ${ }^{3}$ Beijing Jiaotong University, Beijing, China \\ ${ }^{4}$ North China Electric Power University, Beijing, China \\ a18600365587@126.com, b15901168062@163.com, chanwanjiao2007@sina.com, \\ d413501559@qq.com
}

Keywords: building air conditioning, intelligent use of electricity, demand response, strategy, regulate and control

Abstract. For problems in the process of building central air conditioning system to participate in demand response, including non-uniform communication protocol, non-uniform information model and lack of demand response strategy, we put forward the thinking and concrete scheme of research and development of the central air conditioning system automated demand response terminal. It includes the terminal physical architecture, functional architecture, application process and key technology and so on. The terminal has been used demand response pilot project in Shanghai, Jiangsu, Beijing. This application not only promoted the application of domestic demand response business, but also was beneficial to postpone investment growth of the power generation, transmission and substation engineering, promoted the overall efficiency of power system, and promoted the overall improvement of energy efficiency.

\section{Introduction}

In hot summer, the central air conditioning load is too high to result the phenomenon of grid peak and shortage of electric in local areas, especially in the eastern coastal areas ${ }^{[1]}$. Implement demand response for central air conditioning system, on the one hand, can alleviate the influence that is from grid peak on safe and reliable operation of power grids; on the other hand can improve the operation efficiency of the power system and reduce unnecessary waste of resources ${ }^{[2]}$.

However, when the central air conditioning system to participate in demand response, the system cannot automatically analyze requirements demand response from the server response signal, and also can't according to the demand response signal generated directly control command for air conditioning units or units inside air conditioning, such as host, pump, fan, etc. Therefore, design a kind of automatic demand response terminal and its application methods for the central air conditioning system is helpful to support the central air conditioning system to participate in demand response business, save electricity for the central air conditioning system users, promote economic efficient operation in power system, is an important technology to ease energy and environmental problems.

\section{Terminal Physical Architecture}

As shown in Figure 1, central air conditioning system demand response terminal automatically consists of microprocessor module, data storage module, human-computer interaction module, communication module, security module, isolation module, interface module, real-time clock, watchdog and power management module and shell. All the above-mentioned modules are set in the shell and the shell internal coating has electromagnetic shielding layer. 


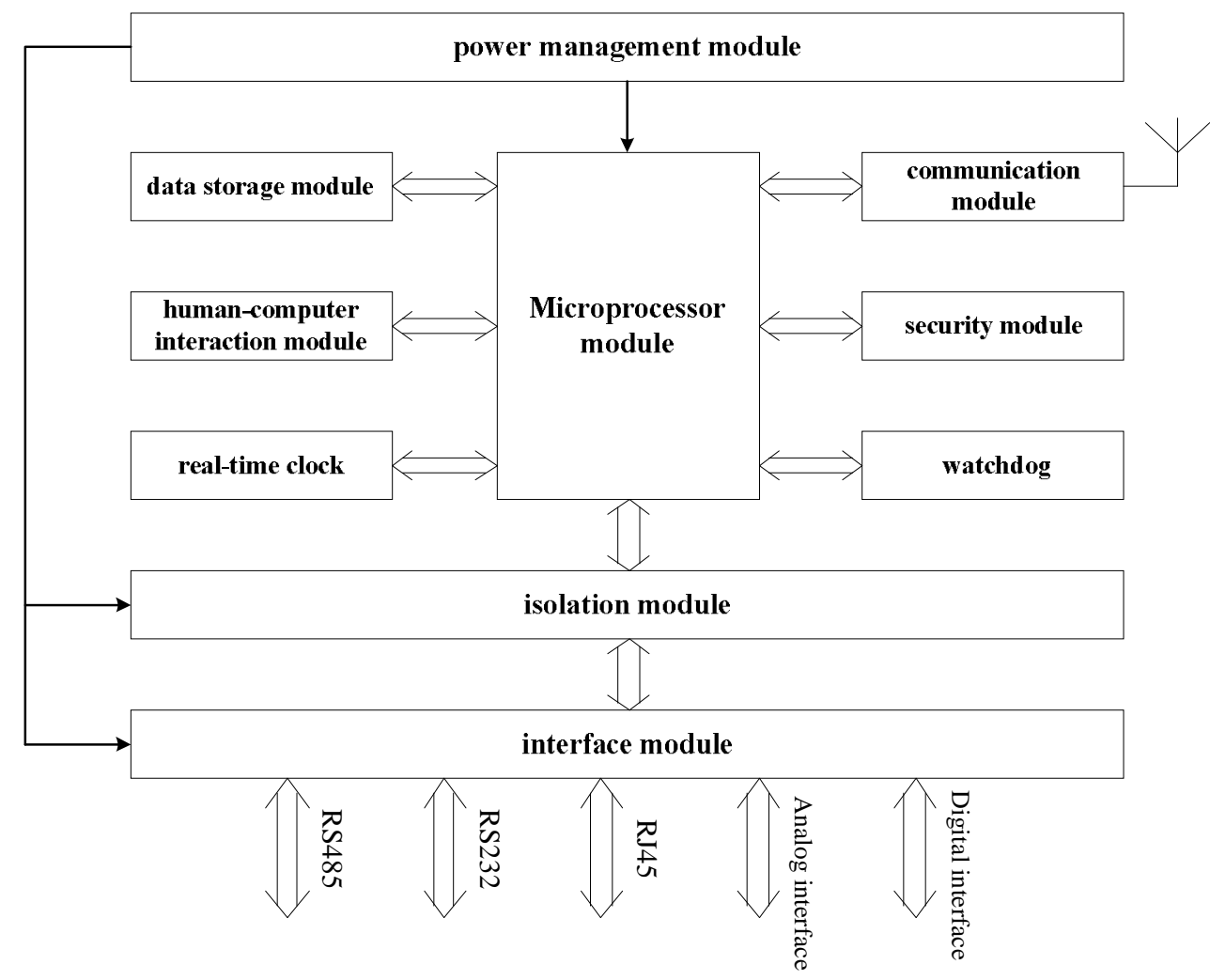

Fig. 1 Terminal physical architecture

Among them: the microprocessor module is the core device and is responsible for interactive information of data storage module, human-computer interaction module and itself. It also executes task process, such as information receiving, information sending, information analysis, strategy implementation, and control commands sending. Data storage module is used to store operation parameters and historical data information and also has built-in demand response strategy library, central air conditioning control operation strategy library which can be update when needed. Human-computer interaction module is used to realize information interaction of users and demand response device. Communication module is responsible for contecting to demand response server supporting cable, wireless or hybrid network communication mode. Security module encrypts sensitive data, such as device configuration information, event information demand response and measurement information, preventing information from being malicious tampered, and verifies the validity of information sources. The solation module isolates other equipment at the scene of the electric power users from itself to reduce device interference and improve the stability. Interface module is used to realize communication between demand response equipment and central air conditioning system, send control instruction, collect and monitor central running status parameters of air conditioning system, and at the same time connects to collectors of electric parameters, environmental parameters and receives electrical parameters and environmental parameters of the running central air conditioning system. Real time clock provides device with accurate system time, and ensure that time will not lost after power. Watchdog prevents device from crash and losing the ability to monitor. Power management module is responsible for the management and allocation of device power supply. The shell and the shell internal coating has electromagnetic shielding layer which is polymer layer.

\section{Terminal Function}

\section{Communications functions}

As shown in Figure 2, automated demand response terminals installed in the field where the central air conditioning system is. For the upper, the device communicate with demand response server through a communication network; For the lower, use RS485, RS232 or RJ45 to realize 
communication with central air conditioning system control module, electric parameters collectors and environmental parameters collectors.

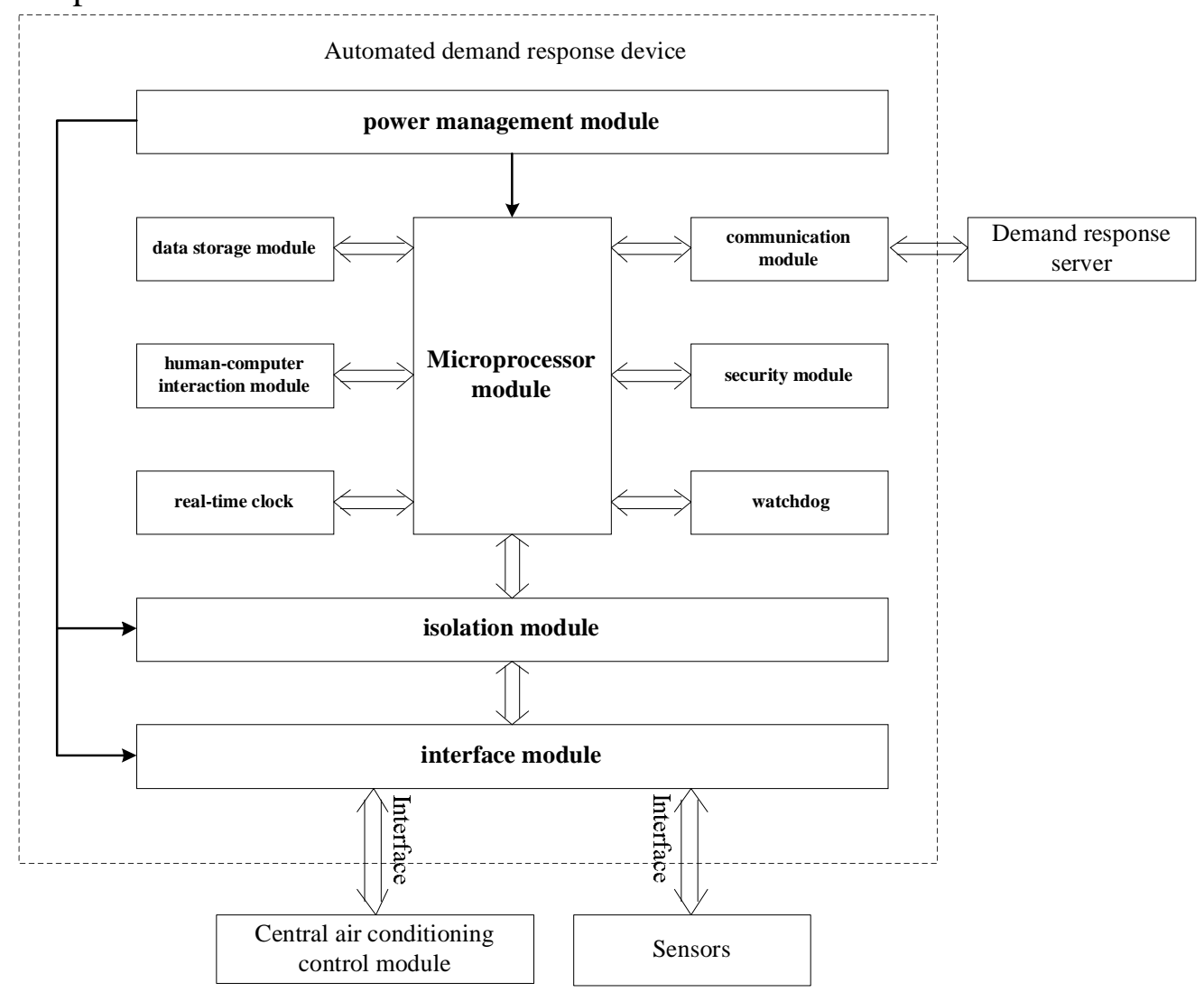

2 Data acquisition function

Fig. 2 Communications functions

Data acquisition function mainly includes electrical parameters, running status and environment parameter. Electric parameter acquisition mainly involved parameters such as voltage, current and power. Running state parameter acquisition mainly involves switch machine state (on, off), the running state of the air (on, off), the electric heating running state (on, off), the operation mode (intelligence, refrigeration, heating, moisture, air supply, etc.), operating temperature, operating wind speed. The environment parameter acquisition mainly relates to indoor and outdoor temperature and humidity.

3 Control function

Terminal has a built-in demand response strategy library. Using the related demand response strategy in the library, the terminal can select appropriate demand response strategy, according to the demand response signal which is received and decoded from demand response server, including response level andresponse capacity. The library can update according to demand response server information.

As shown in Figure 3, the control function also includes central air conditioning control operation strategy library, central air conditioning system parameters selected automatically in real time and generating control commands for central air conditioning system according to response level and response capacity. Control strategies can aim at different units of central air conditioning systems or related components of a single unit ${ }^{[3]}$. The strategy can take joint control for some units to on/off a few units or adjust some units operating power. It can also take control for components of central air conditioning system, aiming at control air conditioning host, pump and fan speed or running state. 


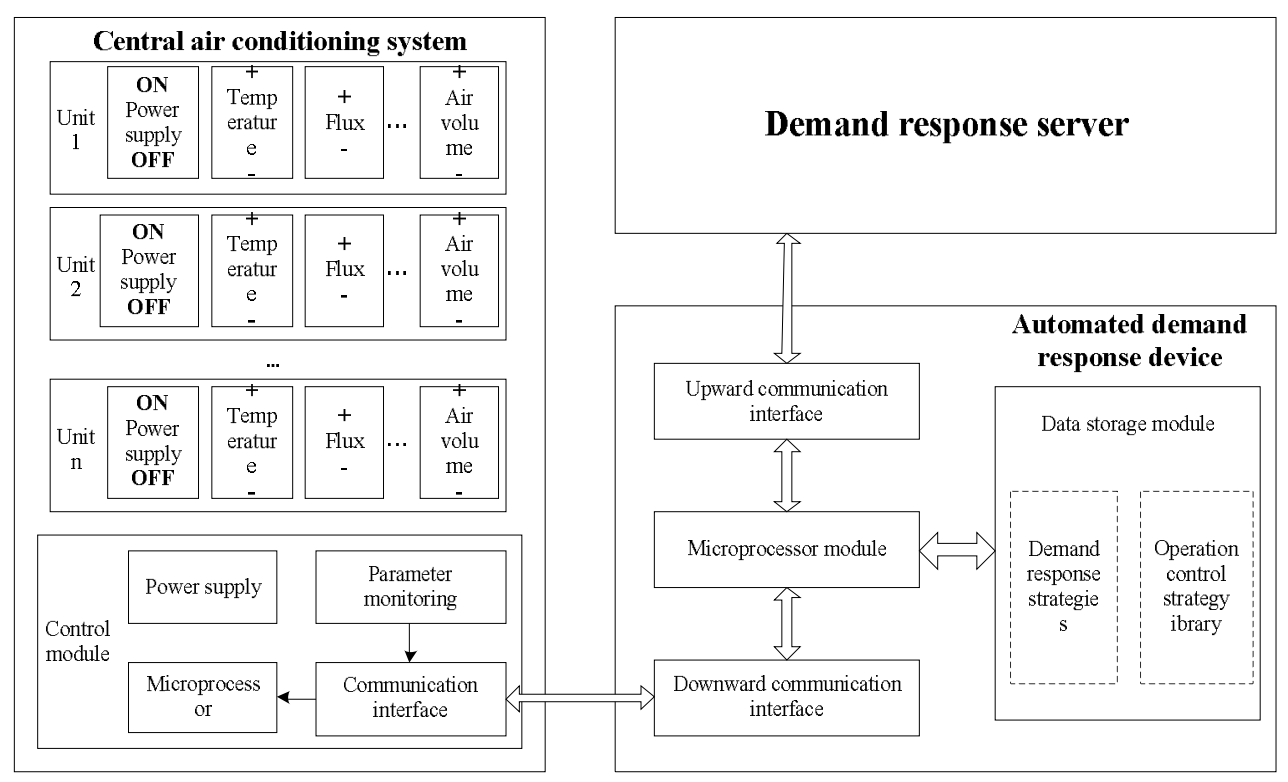

Fig. 3 Control function

4 Safety protection function

The teminal can encrypt sensitive data, such as itself configuration information, event information demand response and measurement information, preventing information from being malicious tampered, and verifies the validity of information sources.

\section{Terminal Application Process}

As shown in Figure 4, in the business of demand response, implement intelligent, automated load control for central air conditioning system. Specific process is as follows:

Firstly, automated demand response terminal through a communication network receives demand response events from the grid side demand response server, according to decorded information such as load, price changes, matching the corresponding demand response strategy.

Secondly, According to the corresponding response demand strategy, integrating current electrical, operation and environmental parameters of central air conditioning system, determines response level and response capacity, and sends feedback signal to the grid side demand response server;

Thirdly, according to the receiving feedback signal, power side demand response server sends confirmation signal to automated demand response terminal.

Finally, according to the established response level and response capacity, based on the built-in central air conditioning control operation strategy library, automatic demand response terminal generates control commands for central air conditioning system. 


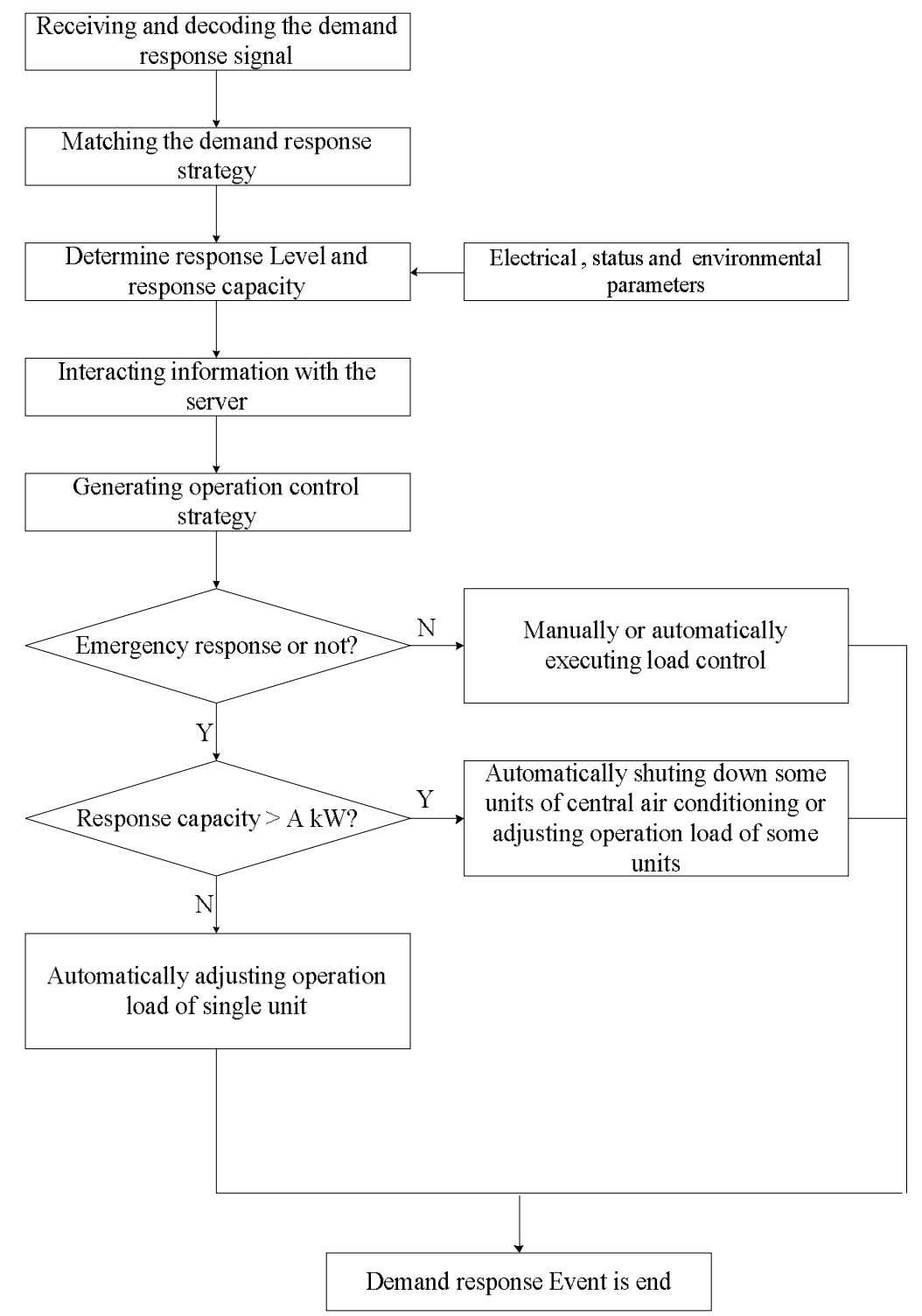

Fig. 4 Terminal application process

\section{Application Effect}

From the aspect of taking part in automated demand response business, automated demand response terminal for central air conditioning system can replace human labor to automatically realize load control of central air conditioning system, therfore avoiding the unnecessary human resources expenditure. The terminal has a built-in demand response strategy library. The stratefy is based on fuzzy algorithm, and can automatically decided to participate in the plan of automated demand response, combined with its own operation parameters and environmental parameters, according to the demand response event information from the grid side demand response $\operatorname{server}^{[4,5]}$, so that the execution efficiency of automated demand response business is improved. The terminal also has a built-in central air conditioning control operation strategy library. The stratefy is based on fuzzy algorithm, according to the response plan information from the grid side demand response server, executes automatic load control for central air conditioning system following the schedule.

The automated demand response terminal takes minimum environmental conditions of industrial and commercial users as constraint standard. When executing operation control strategy, the teminal takes joint control for some different units, or coordinated control for different parts in a single unit, thereby enssuring indoor environmental conditions can meet the demand of industrial and commercial users. 


\section{References}

[1] Ai Zhen, Huang Yuqi, Yang Xi. Air conditioning load control technology research based on demand response[C]. Power communication management and smart grid communication technology BBS, 2013.

[2] Zhou Zhaomao. The progress and practice of the demand response[J]. Power Supply, 2014(3):16-20.

[3] Yue Ming. Systemic solutions and application of building central air conditioning load management[J]. POWER DSM, 2016, 18(3).

[4] Huang Dong. Application of Fuzzy Control to Central Air-conditioning System[J]. Building Electricity, 2013, 32(1):54-59.

[5] Shi Quansheng, Liu Ye. The smart grid and demand response[J]. Public Power, 2013(5). 